\title{
CRYOGENICS FOR CERN EXPERIMENTS. PAST, PRESENT AND FUTURE.
}

Johan Bremer, Jean Pierre Dauvergne, Dimitri Delikaris, Nicolas Delruelle, Friedrich Haug, Gerhard Kesseler, Giorgio Passardi, Jean Michel Rieubland, Johann Tischhauser.

European Organisation for Nuclear Research, CERN, CH-1211 Geneva 23.

\begin{abstract}
Use of cryogenics at CERN was originated (in the 1960s) by bubble chambers and the associated s.c. solenoids. Complex cryoplants were installed to provide cooling at $\mathrm{LH}_{2}$ and $\mathrm{LHe}$ temperatures. Continuity (in the 1970s) in He cryogenics for experiments was provided by spectrometer magnets for fixed target physics of the SPS accelerator. More recently (in the 1980s), large "particle-transparent" s.c. solenoids for collider experiments (LEP) have been built demanding new cryoplants. The LHC experiments (in the 2000s) will continue the tradition with s.c. dipoles (ALICE and LHC-B), solenoids (CMS, ATLAS) and toroids (ATLAS) of unusual size. Cryogenics for experiments using noble liquids follows the same trend since the development (in the 1970s) of the first shower LAr detectors. A LKr calorimeter (about $10 \mathrm{~m}^{3}$ ) will be operated in 1996 and the ATLAS experiment foresees a set of three huge LAr calorimeters (almost $90 \mathrm{~m}^{3}$ total volume of liquid) to be installed underground.
\end{abstract}

\section{INTRODUCTION}

Cryogenics for CERN accelerators, limited to some application in the past, has expanded considerably following the recent development of superconducting (s.c.) accelerating cavities and high field bending magnets. Cryoplants for a total entropy equivalent capacity of $48 \mathrm{~kW} / 4.5 \mathrm{~K}$ are in operation for the LEP2 phase of the Large Electron-Positron Collider (LEP). A factor three increase of the equivalent capacity is foreseen for the Large Hadron Collider (LHC) [1] leading to the world's largest cryosystem.

Cryogenics for experiments behaves differently and indicates a rather continuous use of cooling capacity (Fig.1) during the entire CERN evolution. Future outlook for the LHC experiments shows that the need of cryogenics will extend well beyond the year 2005 with an increase of the expected total entropy equivalent load up to $15 \mathrm{~kW} / 4.5 \mathrm{~K}$.

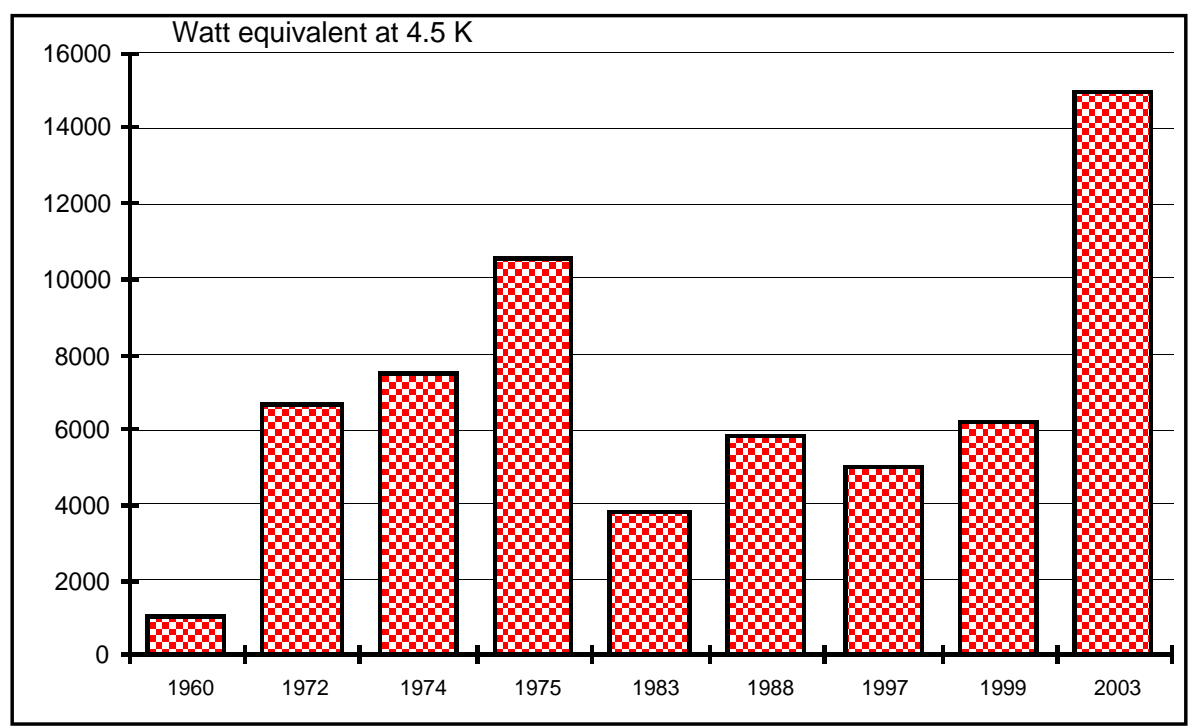

Figure 1 Evolution of cooling capacity for CERN experiments 
Cryogenics at CERN was originated by bubble chambers using several fluids: $\mathrm{LH}_{2}, \mathrm{LD}_{2}, \mathrm{LNe}$ and their binary mixtures. Sensitive volumes ranged from $35 \mathrm{~m}^{3}$ (BEBC) down to a few litres of the rapid cycling $(30 \mathrm{~Hz})$ chambers (LEBC) [2, 3]. Particle momentum analysis requested s.c. magnets adding LHe refrigeration. The complexity (as a single unit) of the cryogenic system of BEBC is still unequalled at CERN and only the LHC will lead to cryosystems of comparable diversification.

Bubble chambers magnets were He bath cooled, a technique requiring large quantities of liquid with considerable safety and inventory drawbacks. BEBC had a $3.5 \mathrm{~T}, 4.7 \mathrm{~m}$ inner diameter magnet with a stored energy of $750 \mathrm{MJ}$. The cold mass $(250 \mathrm{t})$ was immersed in a $20 \mathrm{~m}^{3}$ bath. Refrigeration of the chambers was carried out by two-phase flow of $\mathrm{LH}_{2}$ providing isothermal cooling in the range 22 to $30 \mathrm{~K}$. Only the rapid cycling chamber $\left(\mathrm{RCBC}, 200\right.$ litres of $\left.\mathrm{LH}_{2}\right)$ used gaseous He refrigeration for the internal heat exchanger(s). BEBC complex was cooled by a combined $\mathrm{He} / \mathrm{H}_{2}$ refrigerator concentrating most of the capacity (turbines) on the $\mathrm{He}$ side pre-cooling the $\mathrm{H}_{2}$ circuit at $42 \mathrm{~K}$. In the combined mode of operation $(4.5 \mathrm{~K}$ and $22 \mathrm{~K})$, the system had an entropy equivalent capacity of $6.7 \mathrm{~kW} / 4.5 \mathrm{~K}$. The most challenging additional experimental facility of BEBC was a $3 \mathrm{~m}^{3} \mathrm{LH}_{2}$ track sensitive target (TST). The TST, immersed in the chamber filled with $\mathrm{Ne} / \mathrm{H}_{2}$ mixture $(75 \%$ mole $\mathrm{Ne})$, was made of a thermoplastic polycarbonate having the desired mechanical properties at low temperatures to allow pressure cycling in synchronisation with the chamber [3].

Studies of interaction of incident beams with simple and high density nuclei led to the construction of $\mathrm{LH}_{2}$ and $\mathrm{LD}_{2}$ non sensitive targets with volumes ranging again from large $\left(35 \mathrm{~m}^{3}\right)$ down to a few litres. Common characteristics of the cryogenic facilities were condensation in gravity based systems by means of cryocoolers (both Stirling and Gifford-McMahon cycles).

Cryogenics at $\mathrm{LN}_{2}$ temperatures followed the development of calorimeters utilising the principle of sampling ionisation chambers filled with LAr. Several calorimeters (typical LAr volumes 2 to $4 \mathrm{~m}^{3}$ ) were built for proton-proton experiments in the Intersecting Storage Ring (ISR) [4, 5] and for fixed target physics of the Super Proton Synchrotron (SPS) [6].

They used a variety of cooling methods: forced flow of LAr kept sub-cooled to prevent boiling inside the sensitive volume, gas re-condensation by $\mathrm{LN}_{2}$ cooled heat exchanger(s) integrated at the top of the cryostat, re-condensation of gas in an heat exchanger located outside the cryostat and immersed in a bath of $\mathrm{LN}_{2}$ maintained at constant pressure by cryogenerator(s) (Stirling cycle).

At the exception of a few small $\mathrm{LH}_{2}$ targets, all these facilities have been dismantled.

\section{PRESENT SITUATION}

In parallel or following the bubble chambers, SPS fixed target and p-p ISR physics required the construction of 6 s.c. magnets for particle momentum analysis (Table 1).

Table 1 Superconducting particle spectrometers

\begin{tabular}{|c|c|c|c|c|c|c|c|c|c|}
\hline \multirow[t]{2}{*}{ MAGNET: } & BEBC & OMEGA & ISR & SPS/NA & SPS/NA & SPS/NA & EHS & ALEPH & DELPHI \\
\hline & & & & Vertex & Vertex & Dipole & $\mathrm{RCBC}$ & & \\
\hline $\begin{array}{l}\text { Field: } \\
\mathrm{T}\end{array}$ & 3.5 & 1.8 & 1.5 & 1.5 & 1.5 & 1.9 & 3 & 1.5 & 1.2 \\
\hline $\begin{array}{l}\text { Stored } \\
\text { energy : } \\
\text { MJ }\end{array}$ & 750 & 50 & 3 & $\leq 50$ & $\leq 50$ & 20 & 55 & 130 & 120 \\
\hline $\begin{array}{l}\text { Cold mass: } \\
\mathrm{t}\end{array}$ & 250 & 50 & 2 & 25 & 25 & 40 & 24 & 30 & 30 \\
\hline $\begin{array}{l}\text { Cooling } \\
\text { principle }\end{array}$ & $\begin{array}{l}\text { Helium } \\
\text { bath }\end{array}$ & $\begin{array}{c}\text { Super- } \\
\text { critical } \\
\text { He with } \\
\text { recooling }\end{array}$ & $\begin{array}{l}\text { Helium } \\
\text { bath }\end{array}$ & \multicolumn{3}{|c|}{$\begin{array}{l}\text { Helium two-phase } \\
\text { circulated by pumps }\end{array}$} & $\begin{array}{c}\text { Helium } \\
\text { bath }\end{array}$ & \multicolumn{2}{|c|}{$\begin{array}{l}\text { Indirect } \\
\text { He two-phase } \\
\text { mosyphon Pumps }\end{array}$} \\
\hline Institute & CERN & CERN & CERN & CERN & CERN & CERN & Saclay & Saclay & Ral \\
\hline Operation & no & end 96 & no & yes & yes & yes & yes & yes & yes \\
\hline
\end{tabular}


Bath cooling was progressively abandoned in favour of forced flow of He in hollow conductors [7], simplifying the cryogenic system and the cryostats. The magnet for OMEGA experiment [8] uses supercritical He flow supplied by the refrigerator with periodic bath re-cooling. A concept applied or foreseen for long string of accelerator s.c. magnets (RHIC at BNL and SSC). Forced flow of two-phase $\mathrm{He}$, providing isothermal cooling, was more recently introduced [9]. Possible flow instabilities were overcome by assuring a low outlet vapour content and high flow rate per unit area by means of reciprocating LHe pumps [10]. This solution will be adopted by the s.c. magnets of the ATLAS experiment of LHC. Following the physics program, the use and location of most of the 6 magnets have frequently changed. The layout flexibility was achieved thanks to the distributed cryosystem of the SPS North Area consisting of 7 cold boxes (total entropy equivalent capacity $3 \mathrm{~kW} / 4.5 \mathrm{~K}$ ) having in common a central compression station ( 6 reciprocating +1 screw compressors) [11]. A further advantage offered by this distributed system is the operational flexibility in matching the different time schedule.

One of the North Area cryoplants is used by a new polarised target experiment. The target containing 5 litres of matter is made of irradiated ammonia kept at $50 \mathrm{mK}$ and immersed in a field of $2.5 \mathrm{~T}$ to provide the same spin direction of all the protons. This experiment continues the tradition of high energy physics experiments at very low temperatures initiated with the development of dilution refrigerators [12].

Of the four experiments of LEP in operation since 1989, two (ALEPH and DELPHI) required s.c. "particle-transparent" high field large solenoids (Table 1) for momentum resolution of the secondary particles produced in the collisions.

For both solenoids, indirect cooling method was adopted and He flow with low vapour content at coils outlet was provided by two totally different systems. ALEPH used an innovative thermosyphon concept [13] whereas DELPHI preferred an active system using LHe pumps. Refrigeration is provided by two identical cryoplants [14] for a total entropy equivalent of $2 \mathrm{~kW} / 4.5 \mathrm{~K}$. Main characteristics were the adoption, for the first time at CERN, of oil-lubricated screw compressors and of highly automated control system. Moreover, each cryoplant is capable of matching different thermal situations imposed by the operation in parallel of the low $ß$ quadrupoles associated to the experiments.

The use of noble liquids is continuing for a novel SPS experiment (first operation planned in 1996) based on a $\mathrm{LKr}$ calorimeter (total volume of liquid about $10 \mathrm{~m}^{3}$ ) [15].

To minimise risks of krypton losses because of lack of refrigeration, both the storage dewar and the calorimeter have two separate heat exchangers cooled by $\mathrm{LN}_{2}$ : one external and the other internal. The first one re-condenses the evaporated gas via an intermediate bath of LAr and feeds by gravity the corresponding vessel. The second one exchanges heat directly with krypton and will be used only for emergency situations. The intermediate LAr bath allows to operate the $\mathrm{LN}_{2}$ system at about $0.5 \mathrm{MPa}$ because Ar has a triple point $(84 \mathrm{~K})$ lower than $\mathrm{Kr}(116 \mathrm{~K})$. Both gaseous (in the re-condensing circuit) and liquid (via a circulation pump) on-line purification have been implemented.

\section{FUTURE PERSPECTIVES}

The LHC project foresees four new experiments (Fig.2).

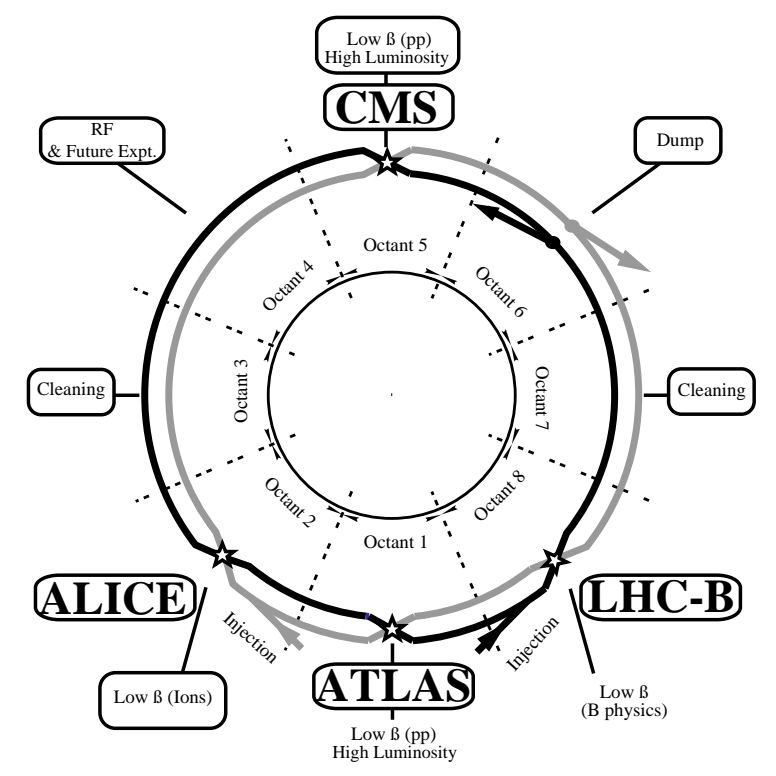

Figure 2 LHC Layout 
ATLAS and CMS $[16,17]$, recently approved, include large cryogenic components. ALICE and LHC-B, in the proposal stage, envisage s.c. solutions respectively for the magnets of a muon arm and for the spectrometer analysing particles in a small solid angle forward direction.

ATLAS is the most demanding in term of cryogenics [18], since the detector is based on three toroidal s.c. magnets, three LAr calorimeters and one central solenoid surrounding the inner detector. A system of s.c. toroidal magnets is justified by the high quality of the muon spectrometer, one of the guiding design criteria of ATLAS. Each toroid consists of eight coils. They are made of flat pancakes in a racetrack configuration and are assembled radially and symmetrically around the beam axis. The barrel s.c. toroid extends over $25 \mathrm{~m}$ and has an i./o. diameter of 9.4 and $20 \mathrm{~m}$. Each of the two end-caps s.c. toroid has an axial length of $5 \mathrm{~m}$ and an i./o. diameter of $1.6 \mathrm{~m}$ and $10.6 \mathrm{~m}$. The $2 \mathrm{~T}$ solenoid $(5.3 \mathrm{~m}$ long, inner diameter $2.3 \mathrm{~m}$ ) is positioned in front of the barrel calorimeter and is integrated into its vacuum vessel to avoid degrading detector performance because of additional walls. The barrel part of the LAr calorimeter (a $6.8 \mathrm{~m}$ long, 2.3 and $4.5 \mathrm{~m}$ i./o. diameter cryostat) is an e.m. "accordion" [16]. The LAr endcaps combine in the same cryostat an e.m. "accordion" and an hadronic calorimeter with flat absorber plates. Each of them extends over $3.2 \mathrm{~m}$ and has an external diameter of $4.5 \mathrm{~m}$. The total cold mass of the calorimeters is $570 \mathrm{t}$.

CMS is based on a solenoid (13 m long, inner diameter of $5.9 \mathrm{~m}$ ) with an uniform field of $4 \mathrm{~T}$. The design is such that the associated muon spectrometer uses a single magnet simplifying the detector design. The required number of ampere-turns introduces new important features to resist the greater magnetic forces and carry the higher current: a four layer winding as opposed to one or two layers in most coils and a larger and more complex reinforced conductor.

ATLAS and CMS s.c. magnets have in common He indirect cooling method and enthalpy stabilisation using high purity aluminium. CMS solenoid will be cooled by a thermosyphon system whilst the complex geometry of ATLAS toroids imposes the use of circulating pumps [19] to ensure hydraulic stability of the two-phase He. In the ATLAS solenoid, liquid excess in coils circuit will be provided directly by the refrigerator without the use of pumps [20].

The very large magnet cold masses and e.m. stored energies respectively 570 t, 1.6 GJ for ATLAS and $260 \mathrm{t}, 2.5 \mathrm{GJ}$ for CMS add extra cooling requirements to the basic thermal load (Table 2).

Table 2 Cooling capacities for LHC Experiments

\begin{tabular}{|l|c|c|c|c|c|}
\hline \multirow{2}{*}{ LHC Experiment } & \multicolumn{2}{|c|}{ ATLAS } & CMS & ALICE & LHC-B \\
\cline { 2 - 6 } & $\begin{array}{l}\text { Toroids and } \\
\text { solenoid }\end{array}$ & $\begin{array}{l}\text { Liquid argon } \\
\text { calorimeters }\end{array}$ & Solenoid & Dipole & Dipole \\
\hline Isothermal load at 89.3 K: watt & - & 25000 & - & - & - \\
\hline Load 40 to 80 K: watt & 10600 & - & 3400 & - & - \\
\hline Isothermal load at 4.5 K: watt & 2550 & - & 540 & - & - \\
\hline Liquefaction load: g/s & 10.3 & - & 2.5 & - & - \\
\hline $\begin{array}{l}\text { Total entropy equivalent load } \\
\text { at 4.5 K including } \\
\text { contingency : watt }\end{array}$ & 6000 & - & 1500 & $\sim 1000$ & $\sim 1000$ \\
\hline $\begin{array}{l}\text { Precooling power } \\
300 \text { - 100 K : watt }\end{array}$ & 43000 & 9000 & 18000 & - & - \\
\hline
\end{tabular}

The present design foresees 40 days for cool down from ambient, 4 days (ATLAS) and 12 days (CMS) thermal recovery from post-fast discharge temperatures. To satisfy the 40 days requirement and the envisaged sequential cool down from ambient to $100 \mathrm{~K}$ of ATLAS magnets, a cold box with a separate LN2 cooled pre-cooler are foreseen. Below $100 \mathrm{~K}$, cool down or re-cooling after a fast energy dump will be carried out preferentially in parallel mode. An integrated cryosystem (pre-cooler and main cold box forming a single unit) is proposed for CMS having only one magnet.

The LAr system of ATLAS consists of: a $\mathrm{LN}_{2}$ underground refrigerator $(25 \mathrm{~kW}$ at $89 \mathrm{~K})$ with a back-up of two surface $\mathrm{LN}_{2}$ dewars (each $50 \mathrm{~m}^{3}$ ), a pre-cooling unit, two $50 \mathrm{~m}^{3} \mathrm{LAr}$ tanks linked to calorimeters via a bottom line and a $15 \mathrm{~m}^{3}$ underground $\mathrm{LN}_{2}$ buffer. Furthermore, a complex system of flexible cryogenic transfer lines is being studied to allow movements of the end-caps, for access to internal detectors without disconnecting the pipes. All these facilities should allow operation of calorimeters without any interruption since liquid transfer or re-filling might affect its purity and, 
therefore, calorimeter performance. The underground dewars near the detector will make easier the emptying (total liquid volume of calorimeters is $90 \mathrm{~m}^{3}$ ) in case of large liquid spillage.

Operation of calorimeters requires temperature homogeneity and absence of bubbling through the high voltage gaps of the read-out plates. Internal heat exchangers fed with two-phase $\mathrm{LN}_{2}$ or LAr near or below atmospheric pressure will control the temperature distribution. To prevent boiling, the LAr will be kept sub-cooled at any level by means of an hydrostatic external head obtained by positioning the envisaged liquid expansion volumes two meters above the highest calorimeters points.

The cryogenics for the other two experiments (ALICE, LHC-B) is still in a preliminary phase of definition and expected entropy equivalent capacities for each of them range from 0.5 to $1 \mathrm{~kW} / 4.5 \mathrm{~K}$.

\section{REFERENCES}

1. L.Evans, "The Large Hadron Collider Project", Invited paper to this Conference.

2. F.Birchler et al., "Cryogenic Aspects of the 3.7 m European Bubble Chamber", Proceedings 3th Int. Cryogenic Engineering Conference, pp.244-250.

3. H.Leutz, "Track Sensitive Targets, Hybrid-and Rapid Cycling Chambers", Proceedings Supplements Nuclear Physics B, 36 (1994), pp.203-228.

4. C.W.Fabjian et al., "Iron Liquid-Argon and Uranium Liquid-Argon Calorimeters for Hadron Energy Measurement", Nuclear Instruments and Methods, 141 (1977), pp. 61-80.

5. J.H.Cobb et al., "A large Liquid-Argon Shower Detector for an ISR Experiment", Nuclear Instruments and Methods, 158 (1979), pp. 93-110.

6. H.Burckardt et al., "The Beam and Detector for a High Precision Measurement of CP Violation in Neutral-Kaon Decays", Nuclear Instruments and Methods in Physics Research, A268 (1988), pp.116143.

7. M.Morpurgo, "A Superconducting Solenoid Cooled by Forced Circulation of Supercritical Helium", CERN report Nuclear Physics Division, 69-25 (September 1969).

8. M.Morpurgo, "The Design of the Superconducting Magnet for the Omega Project", Particle Accelerators, 1 (1970), pp. 255-263.

9. M.Morpurgo, "A Large Superconducting Dipole Cooled by Forced Circulation of two Phase Helium", Cryogenics, (July 1979), pp.411-414.

10. M.Morpurgo, "Design and Construction of a Pump for Liquid Helium", Cryogenics, (February 1977), pp.91-93.

11. D.Delikaris, J.P.Dauvergne, F.Haug, "Technical Analysis and Statistics from Long Term Helium Cryoplant Operation with Experimental Superconducting Magnets", Paper submitted to this Conference.

12. T.O.Niniikoski, "Dilution Refrigeration: New Concepts", Proceeding 6th Int. Cryogenic Engineering Conference, (1976) Grenoble, pp. 102-111.

13. J.C.Lottin and R.Duthil, "Aleph Solenoid Cryogenic System", Proceedings 12th Int. Cryogenic Engineering Conference", (1988), pp.117-121.

14. J.P.Dauvergne et al., "Helium Cryogenics at LEP Experimental Areas", Advances in Cryogenic Engineering, 35b (1989), pp.901-908.

15. NA48 collaboration, "The NA48 Liquid Krypton Calorimeter", Nuclear Instruments and Methods in Physics Research, A316 (1992), pp.1-7.

16. ATLAS collaboration, "Atlas Technical Proposal", CERN/LHCC 94-43, (1994) Geneva.

17. CMS collaboration, "CMS Technical Proposal", CERN/LHCC 94-38, (1994) Geneva.

18. F.Haug, J.P.Dauvergne, G.Passardi, D.Cragg, C.Cure, C.Mayri, A.Yamamoto, "Refrigeration system for the Atlas Experiment", Paper submitted to this Conference.

19. C.Mayri, C.Cure, R.Duthil, D.Cragg, F.Haug, G.Passardi, "Barrel Toroid Cryogenic System for Atlas detector", Paper submitted to this conference.

20. K.Tanaka, A.Yamamoto, Y.Doi, T.Haruyama, T.Kondo, Y.Makida, "Cryogenic Design of the Atlas Thin Superconducting Solenoid Magnet", Paper submitted to this conference. 